\section{Eric SARTORI}

LE SOCIALISME D'AUGUSTE COMTE. AIMER, PENSER, AGIR AU XXI. París: L'Harmattan. 2012. 166 pp.

El objetivo de este libro es redescubrir a Comte como autor sociológico actual y darle el espacio que se merece en la historia del pensamiento sociológico. Muchas veces citado en los manuales de sociología pero casi nunca leído, por considerarlo poco actual, al contrario que otros clásicos como Durkheim o Weber que, sin embargo, siguen condicionando e influyendo en la doctrina sociológica contemporánea, Comte no es sólo el nombre de una estatua del Barrio Latino o de una calle de París, y tampoco se puede recordar sólo por la frase "orden y progreso" que aparece en la bandera de Brasil.

Comte es también el padre del positivismo, la única corriente ideológica y filosófica que no ha originado regímenes dictatoriales en el siglo XIX-XX. Es, además, un pensador menos conservador de lo que siempre se ha creído, un autor liberal que sin embargo no llegó a apoyar el liberalismo de Adam Smith y que a la vez rechazó rotundamente el marxismo y cualquier ideología revolucionaria y palingenésica. Un precursor de la tercera vía entre liberalismo y socialismo, entre el libre mercado y el dominio del Estado. Comte en este sentido se inscribe en un panteón que reúne en una única grande Iglesia pensadores recientes y lejanos en el tiempo, como Bobbio, Bernstein y el propio Durkheim, una ideología que tanto se necesitaría hoy día para superar la lucha dicotómica entre derecha liberal e izquierda estatalista. En esto resiste la actualidad de Comte, y por eso hay que volver a leerlo, después de haber ganado las resistencias y los prejuicios contra un autor considerado demasiadas veces como ingenuo, pedante, y culpable de haber sobrecar- gado de responsabilidades la sociología, cuando la definió como "la nueva ciencia de la humanidad", que habría sustituido a la religión.

Por último, el autor recuerda que también Raymond Aron dedicó cierto espacio al pensamiento de Comte, definiéndolo como el sociólogo de la era industrial.

\section{G. TARDIVO \\ U. Rey Juan Carlos (Madrid)}

\section{John W. CRESWELL}

A CONCISE INTRODUCTION TO MIXED METHODS RESEARCH. Thousand Oaks (CA): Sage. 2014. 152 pp.

Recientemente ha visto la luz este notable trabajo de J. W. Creswell, que abunda con él en la línea de estudios metodológicos sobre modos mixtos de investigación social que este profesor de la Universidad de NebraskaLincoln ha venido desarrollando en los últimos años con considerable impacto de difusión. $\mathrm{Su}$ anterior obra, Designing and conducting mixed methods research (2011), escrita en colaboración con la profesora Vicki Plano Clark, ya se había convertido en una referencia obligada para los investigadores sociales inclinados al enfoque multimétodo. Los modos mixtos en la investigación social no son en sentido estricto una novedad metodológica, ya que los investigadores han recogido y utilizado datos cuantitativos y cualitativos, en variadas combinaciones, desde hace ya bastante tiempo: ahí están, por ejemplo, las contribuciones de Campbell y Fiske (1959) sobre el uso combinado de múltiples métodos cuantitativos, la de Sieber (1973) utilizando conjuntamente encuestas y entrevistas, la de Jick (1979) en relación con los procedimientos de triangulación mediante datos cuantitativos y cualitativos, o la de Cook y Reichardt (1979) con sus diez vías de articular las perspectivas cualitativa y cuantitativa. 
Sin embargo, entrelazar ambos tipos de datos, cualitativos y cuantitativos, como un diseño de investigación diferenciado, tal como Creswell lo propone, sí constituye una innovación metodológica, que inició su andadura hace apenas un decenio.

Hay dos elementos clave en este libro que le proporcionan su singularidad metodológica: el original planteamiento de las fases del proceso de investigación con modos mixtos y la presentación de cuatro diseños concretos para combinar distintos modos de investigación. Tanto uno como otro elemento descansan sobre la experiencia de investigaciones empíricas del autor, confiriendo a la obra un carácter práctico y realista que muchos lectores agradecerán. Una de las virtudes que posee la forma en que se plantean las fases de una investigación con modos mixtos es que no se ofrece como una propuesta metodológica cerrada, sino como una visión general de la estructura procedimental apropiada para investigar con esta metodología, que permite variaciones y versiones adaptadas a los propósitos de cualquier investigador.

El libro está compuesto por diez capítulos que abarcan la práctica totalidad de las cuestiones involucradas en la investigación social con modos mixtos. El primero de ellos trata de las características básicas de la investigación con esta metodología, abordando los temas fundamentales para su comprensión, tales como la clarificación terminológica y conceptual, la naturaleza de los datos cuantitativos y cualitativos, las posibilidades de combinarlos (fusionarlos, conectarlos o imbricarlos) en diversos tipos de investigaciones (simples o múltiples), las respuestas que dan a diferentes problemas de investigación y las posibilidades de aplicación en muy variados fenómenos sociológicos. El segundo capítulo aborda el desarrollo y avance de la metodología desde sus inicios hasta la actualidad, con énfasis en los logros alcanzados en la última década, y examina con cierto detenimiento asuntos capitales como los fundamentos esenciales de las perspectivas cuantitativa y cualitativa, la determinación precisa del problema de investigación y si en efecto la investigación pretendida se puede llevar a cabo usando una metodología de modos mixtos. El tercer capítulo describe los pasos necesarios para diseñar una investigación con esta metodología, utilizando abundantes referencias de casos reales. El cuarto advierte de cuáles son las herramientas que deben emplearse en una investigación social si se quieren usar los modos mixtos y constituye una especie de guía para escoger un tipo concreto de combinación en función del objeto de investigación. El quinto capítulo es ya una introducción práctica a la metodología, mostrando para su mejor comprensión diseños simples y avanzados de su aplicación. El sexto muestra cómo dibujar un diagrama de procedimientos para usar un diseño de modos mixtos y cómo actuar para recoger datos de calidad de diversas clases. El séptimo capítulo versa sobre las maneras de realizar un análisis de los datos obtenidos con esta metodología y sobre cómo llevar a cabo la validación de un diseño de investigación que se sirve de ella. El octavo capítulo aborda la problemática, verdaderamente crítica, de la obtención de muestras de datos de origen metodológico diferente y de su integración en un único cuerpo de información sociológica que se nutre de todas las fuentes utilizadas. El noveno aborda, precisamente, la cuestión de cómo articular informativamente los datos de diferente naturaleza obtenidos con varios métodos y redactar un informe que, trascendida su diversidad de origen, los reúna en un relato técnico-científico. El décimo capítulo, en fin, versa sobre la contribución que suponen los métodos mixtos para la investigación social y de cómo puede evaluarse la calidad de los diseños que se valen de esta metodología.

En definitiva, en este interesante libro se repasan las características básicas de esta propuesta metodológica y se muestra, en aquello 
que es más esencial e importante, el procedimiento apropiado para utilizarla. En tanto que los modos mixtos constituyen una metodología diferenciada, se describen didácticamente cuáles son sus presupuestos fundamentales, conceptuales y empíricos, de tal manera que cualquier lector con una mínima preparación y experiencia en investigación social aplicada pueda familiarizarse rápidamente con el aparato terminológico y técnico que su uso comporta. Es este sentido, es sin duda un acierto recoger en el libro los suficientes casos prácticos, que ilustran paradigmáticamente los usos y posibilidades de esta metodología. El libro supone una defensa del principio de que la combinación de métodos y perspectivas no sólo proporciona al investigador social una mejor comprensión de los fenómenos que le competen que si se emplean por separado, sino que dicha combinación responde mejor a la verdadera naturaleza de los objetos del mundo social, que siempre están compuestos y siempre ofrecen un lado cuantitativo (expresan una pluralidad) y un lado cualitativo (significan algo) si son sociológicamente relevantes. La complejidad de los fenómenos sociales actuales hace que la articulación de procedimientos de investigación multienfoque probablemente sea tan oportuna ahora como pocas veces lo fue antes, y el libro de Creswell ofrece la posibilidad de acercarse a esos procedimientos de forma sencilla, clara y ordenada y constituye un texto técnico de indudable utilidad.

\section{J. L. PALACIOS ACMS (Toledo)}

Laura AROSIO

L'ANALISI DOCUMENTARIA NELLA RICERCA SOCIALE. METODOLOGIA E METODO DAI CLASSICI A INTERNET. Milano: Franco Angeli. 2013. 117 pp.
Este ágil y a la vez interesante libro de metodología es parte de una colección llamada Laboratorio Sociológico, que la editorial italiana Franco Angeli dedica desde 1992, bajo la guía del célebre sociólogo Costantino Cipolla, al tema de la investigación en ciencias sociales. En este texto breve y fácil de leer, de 117 páginas, la investigadora Laura Arosio se dedica a reflexionar sobre el uso y la importancia de los documentos en las investigaciones.

Durante largo tiempo olvidados para dejar espacio a otras técnicas más de moda, el análisis de documentos parece haber vuelto a cobrar cierta importancia en los estudios sociológicos, porque efectivamente varias son las ventajas que ofrece: los documentos dan una visión dinámica y no estática de la realidad, son más económicos que otros medios -sobre todo desde cuando la red de la redes ha facilitado el acceso a muchos de ellos- y en épocas de recortes a la investigación este elemento sin duda alguna se agradece y no puede ser olvidado ni pasado por alto. Además permiten mirar a los fenómenos sociales con una perspectiva interdisciplinar, y, por último, son fundamentales en estudios de carácter comparativo, como recuerda la autora citando a Morlino, Sartori y Smelser, es decir a los autores que han dedicado las reflexiones quizá más interesantes sobre el análisis comparativo. Y, por supuesto, resultan fundamentales cuando los sujetos que queremos estudiar no están al alcance de los investigadores, por lejanía física, cultural, etc.

Recordando brevemente la historia del análisis documental en sociología la autora no puede dejar de citar un estudio pionero desde este punto de vista, referencia para generaciones enteras de sociólogos que han crecido a la sombra de Thomas y de su campesino polaco. Una obra que determinó, como recuerda Laura Arosio, "un momento fundamental para la afirmación en el campo sociológico de los estudios basados en el análisis documental".

El libro termina con una interesante bibliografía con referencias mayoritariamente italia- 
nas, lo cual representa sin duda alguna una novedad respecto a casi todos los manuales de metodología, que presentan sobre todo citas y referencias de autores americanos e ingleses.

Incluso en épocas de neuromarketing, de grupos de discusión y de consumidores camaleónicos, los documentos pueden tener un espacio relevante. Y volver a lo antiguo, es decir, a leer documentos, puede acompañarse felizmente de las demás técnicas de investigación.

G. TARDIVO

U. Rey Juan Carlos (Madrid)

Francisco J. VANACLOCHA BELLVER (dir.)

MARCO LEGAL Y DE GESTIÓN DE LA PROTECCIÓN DE LAS INFRAESTRUCTURAS CRÍTICAS EN ESPAÑA.

Madrid: McGraw-Hill/Interamericana de España. 2013. 176 pp.

Novedoso contenido ofrece al lector este libro, publicado bajo la dirección del profesor F. J. Vanaclocha Bellver, catedrático de Ciencia Política y de la Administración en la Universidad Carlos III de Madrid y director del Instituto de Política y Gobernanza de dicha institución académica; la edición ha sido costeada por la empresa Vinsa Seguridad Alentis, integrada en la ONCE, y cuenta con el beneplácito de las máximas autoridades con competencia en el tema.

Las infraestructuras críticas constituyen el conjunto de servicios esenciales prestados por la infraestructura estratégica (redes, equipos tecnológicos, instalaciones, etc.) para proteger la seguridad física, la salud, el bienestar social de los ciudadanos y la producción económica de un país, de modo que su destrucción es irreemplazable y ocasiona graves perjuicios a la sociedad. Las infraestructuras críticas, y su mantenimiento funcional óptimo, revisten enorme importancia en los últimos años para los individuos y países, envueltos en escenarios nacionales e internacionales cargados de incertidumbre, amenazas y crisis.

Frente a la respuesta defensiva a posteriori como única alternativa, se impone hoy la prevención de riesgos y catástrofes, y una gestión integral para la protección de las estructuras implicadas, lo que requiere un seguimiento continuado y experto de las complejas interacciones que establecen los servicios esenciales en las sociedades actuales. Desde tan avanzada perspectiva, el presente texto se dedica al análisis del sistema de infraestructuras críticas existente en nuestro país, al estudio del contexto político-administrativo y del marco legislativo en que se inserta, y a la explicación y valoración de las planificaciones exigidas para afrontar con éxito la gobernanza de las mismas.

Respecto a la organización de los contenidos, a una breve Presentación realizada por Francisco J. Vanaclocha, siguen tres prólogos, cuyas autorías corresponden, respectivamente, a Francisco Martínez Vázquez, Secretario de Estado de Seguridad; Rafael Catalá Polo, exSecretario de Estado de Infraestructuras, Transportes y Vivienda y actual ministro de Justicia; y Faustino Díez Rodríguez, Director Nacional de Vinsa Seguridad Alentis. En todos ellos se evidencia la trascendencia de las medidas a tomar, y la urgencia y eficacia a la que deben responder.

La parte central del texto contiene tres excelentes estudios. El primero de ellos, "La protección de infraestructuras críticas como espacio emergente de políticas públicas y gobernanza", elaborado por Francisco J. Vanaclocha, analiza los fundamentos de las infraestructuras críticas y su control y gestión en el contexto de las políticas públicas de seguridad y el paradigma de la gobernanza. El segundo estudio, de Blanca Breñosa Sáez de Ibarra, coordinadora del Área normativa de la Vicesecretaría General Técnica del Ministerio del In- 
terior, lleva por título "El proceso de elaboración de la normativa española de protección de las infraestructuras críticas", donde se describe minuciosamente el proceso político, jurídico y administrativo que permitió establecer el marco legal contemplado en la Ley $8 / 2011$, de 28 de abril, por la que se establecen medidas para la protección de las infraestructuras críticas, y el Real Decreto $704 / 2011$, de 20 de mayo, por el que se aprueba el Reglamento de protección de las infraestructuras críticas. El último estudio, "Las políticas de protección de infraestructuras críticas desde una perspectiva comparada. El modelo implantado en España", obra de Fernando J. Sánchez Gómez, Director del Centro Nacional para la Protección de las Infraestructuras Críticas (CNPIC), desarrolla "un estudio comparado de las políticas, tendencias, iniciativas y buenas prácticas puestas en marcha por numerosos países de Europa, Estados Unidos y Canadá, en los últimos años, con sus ventajas, inconvenientes y su grado de implantación. “(p. 62), para pasar a describir y justificar el perfil adoptado por el modelo español.

La tercera y última parte del libro se corresponde con dos anexos. El Anexo I, compuesto por Carlos Carmona Pérez, profesor del Instituto de Política y Gobernanza de la Universidad Carlos III de Madrid, está dedicado a precisar el "Marco conceptual del sistema español de protección de infraestructuras críticas", un útil compendio de términos extraídos de la normativa española sobre el tema. El Anexo II es una recopilación de la legislación vigente sobre infraestructuras críticas españolas, recogiendo también la Directiva 2008/ 114/CE del Consejo de 8 de diciembre de 2008, sobre la materia.

Tanto por la actualidad del tema y de la documentación trabajada, como por el tratamiento riguroso, innovador y divulgativo que de todo ello se hace, el libro constituye un referente en nuestro país; e interesa de modo especial a quienes se ocupan de las áreas relacionadas con la seguridad pública y privada, las nuevas tecnologías de la comunicación, la administración en todos sus niveles, las fuentes de energía y la producción industrial, sin olvidar el ámbito de la sanidad y los sectores relacionados con el bienestar social.

\author{
Mª I. MORÁN MORÁN \\ U. Extremadura (Cáceres)
}

\section{Andrés PIQUERAS}

LA OPCIÓN REFORMISTA: ENTRE EL DESPOTISMO Y LA REVOLUCIÓN. UNA EXPLICACIÓN DEL CAPITALISMO HISTÓRICO A TRAVÉS DE LAS LUCHAS DE CLASE. Madrid: Anthropos. 2014. 224 pp.

El formato de este ensayo se sitúa entre el análisis de la actual crisis socioeconómica global y el manual de política económica. Por ello, tiene la virtud de ofrecerse como un dispositivo de análisis del capitalismo globalizado que puede leerse y manejarse en una doble dirección. El trabajo, tras una explicación histórica de los diferentes estadios del capitalismo y de la lucha de clases, aporta una mirada radical y crítica sobre la situación actual de la dinámica Capital/Trabajo como principal explicación de la crisis del sistema capitalista global. Para ello, la parte final del ensayo está formada por una "coda" o conclusión (anexo II "El drama español") en el que el autor coloca a la sociedad española frente al espejo de su realidad socioeconómica y sus desequilibrios del mercado laboral y del reparto de la riqueza; en definitiva frente a la realidad de unos indicadores socioeconómicos marcados por la creciente desigualdad. Se trata de un ensayo de actualidad extrema, a cuyas conclusiones se llega tras un manual denso y bien organizado que hace repaso a los diferentes estadios del capitalismo, a sus ciclos y a las aproximaciones teóricas que lo hacen aprehensible como sistema en permanente transición y transfor- 
mación.

Decimos que la lectura de este trabajo permite una doble "dirección", porque una vez alcanzadas las conclusiones de estos apartados finales, estas nos impelen a volver sobre el prolijo y meticuloso análisis de la historia del capitalismo a la búsqueda de una respuesta a las preguntas clave que termina planteando, y que en esencia son dos. La primera (p. 173), ¿hasta dónde se puede incrementar la dominación y la explotación de las sociedades sin que haya una reacción masiva? Y la segunda (p. 150), ¿hacia dónde va el sistema capitalista atorado por sus desequilibrios y fracasos, y cuál será la siguiente mutación del sistema capitalista que le permita adecuarse y adaptarse a un nuevo modelo de explotación evitando las consecuencias de sus tensiones internas? Y en relación con esta pregunta, ¿cabe todavía la opción reformista y socialdemócrata, a la vista de la deshumanización de las relaciones Capital/Trabajo como alternativa reguladora del sistema?

La virtud del ensayo es, sin lugar a dudas, formular estas cuestiones claves para conocer la evolución del sistema político económico global actual, y plantear, de manera sólida y justificada estas cuestiones como ejes conductores para entender la situación de las sociedades "centrales" en un contexto global. De manera sistemática, se hace repaso a las dinámicas de reproducción del capital, con sus tensiones y conflictos derivados de la acumulación masiva, la dinámica interestatal, el papel del espacio público y de los mecanismos de regulación, y la dinámica Capital/Trabajo, marcada de manera reiterada por la necesidad, primero de explotación, y luego de "desalarización" de la clase trabajadora en favor del desarrollo tecnológico y computacional. Este trabajo de construcción "cíclica" de la relación Capital/Trabajo, aporta, como una de las herramientas mejor articuladas, un cuadro sinóptico general (p. 187) que organiza y sir- ve de guía para todo el ensayo.

Junto con un exhaustivo repaso de bibliografia, fuentes y citas, este trabajo desgrana un análisis de largo alcance con voluntad de abrir espacios de reflexión sobre la evolución de la crisis actual del modelo económico siguiendo para ello un paradigma crítico y un análisis materialista/marxista. El resultado es, no sólo a las evidencias de unos ciclos históricos de reproducción de los intereses del Capital, sino la existencia, apuntada de manera subterránea durante todo el trabajo, de una coyuntura geopolítica que marca las hojas de ruta del Capital, de los Estados y de la emergente, todavía embrionaria, clase trabajadora organizada a nivel global (p. 182). Es ahí, en el papel de las nuevas economías emergentes, en los diferentes modelos de asalarización vigentes en la economía globalizada y en el papel que juega el denominado "Segundo mundo", donde el texto parece otear posibles cambios en el sistema de regulación y de relaciones Capital/Trabajo. Es también en este conjunto de reflexiones sobre geopolítica-económica donde este ensayo deja abierta una línea de trabajo e investigación que sería interesante abordar para completar un "mapa" de crisis que no termina de perfilar claramente sus relieves y fronteras. Unas fronteras que, por otra parte, serán las que delimiten la posibilidad de que el Capital se someta a la "reforma", a la "revuelta", o siga imperando a caballo del pensamiento único del neoliberalismo.

G. MONTIEL ROIG U. Jaume I (Castellón)

Francisco CEBRIÁN ABELLÁN y Miguel PANADERO MOYA (coords.) CIUDADES MEDIAS. FORMAS DE EXPANSIÓN URBANA. Madrid: Biblioteca Nueva. 2013. 246 pp.

Este libro recoge una interesante investigación llevada a cabo por un equipo de profeso- 
res que trabajan en tres universidades ubicadas en sendos países: Universidad de Castilla-La Mancha (España), Universidad de Varsovia (Polonia) y Universidad de Green Bay (Wisconsin, EE. UU.). Los objetivos del estudio se centran en observar y explicar el proceso de expansión urbana en las ciudades de tamaño medio, europeas y norteamericanas.

El fenómeno de la ciudad difusa, entendido como un proceso de interrelación creciente entre las zonas centrales de las ciudades y las zonas limítrofes, se ha generalizado en los últimos decenios afectando las urbes de todos los tamaños. Sus efectos, más visibles en las ciudades de tamaño grande y mediano, se traducen, entre otros, en el crecimiento poblacional de las localidades próximas, la traslación a las mismas de actividades productivas y de ocio antes inexistentes, el incremento de los flujos de intercambios (socioeconómicos, espaciales), la transformación de los comportamientos tradicionales, y la configuración de asentamientos diversos que retan el principio de sostenibilidad.

Este proceso es estudiado mediante el método comparativo y el estudio de casos. En concreto, los autores analizan y contrastan el origen y el contexto, los rasgos distintivos y similares, y las tendencias de dicho fenómeno en las ciudades de Albacete (España), Green Bay (estado de Wisconsin, Estados Unidos), y Plock y Siedlce (Polonia). Para ello aplican un triple enfoque, integrando variables demográficas, culturales y estructural-funcionales. Las primeras detectan los cambios acontecidos en la población; las segundas inciden en las transformaciones de las pautas laborales y de estilos de vida de los habitantes; y las terceras permiten descubrir los espacios novedosos asignados a las actividades sociales de toda índole y los ejes que orientan su difusión.

Los contenidos se organizan en torno a la investigación realizada, de modo que tras la
Presentación, se ofrecen tres partes diferenciadas. La primera, "El estado de la cuestión", incluye tres capítulos en los que se plantean los rasgos propios de la ciudad difusa en las urbes de tamaño medio de los tres países mencionados, atendiendo a las diferencias marcadas por las peculiaridades históricas y culturales de cada uno. La segunda parte, "Estudios de caso en España, Estados Unidos y Polonia”, analiza con detalle cómo se ha gestado, desarrollado y manifestado el proceso de ciudad difusa en cada una de las cuatro ciudades estudiadas, dedicando un capítulo a cada caso. La tercera parte, "A modo de conclusión. Los modos de la difusión urbana", muestra tanto los factores y procesos comunes a la expansión urbana en las ciudades estudiadas, como la explicación de las variaciones encontradas en la ocurrencia del fenómeno, ya sea porque los orígenes y la consolidación tienen lugar en tiempos distintos y con diferentes grados de intensidad y aceptación.

La mirada científica de los autores esclarece la abundante terminología asociada, frecuentemente de forma equívoca, al concepto de ciudad difusa; también recoge y valora las propuestas teóricas recientes elaboradas sobre las dinámicas espaciales y sociales de las ciudades, así como la polémica en torno a su clasificación según el tamaño.

Se trata, pues, de un volumen bien articulado y de interesante lectura por los cambios sociales, demográficos, culturales, económicos y territoriales que la investigación evidencia. Las conclusiones apuntan, además, a procesos y factores compartidos por ciudades con distinta trayectoria histórica y cultural, facilitando la vivencia de un continuum rural-urbano que trasciende los límites restrictivos de lo local y abre a una dimensión más general.

M I. MORÁN MORÁN U. Extremadura (Cáceres) 
Alfonso ORTEGA GIMÉNEZ (coord.)

GESTIÓN MUNICIPAL DE LA DIVERSIDAD E INTEGRACIÓN DE LA POBLACIÓN INMIGRANTE: PAUTAS PARA LA CORRECTA ELABORACIÓN, GESTIÓN Y SEGUIMIENTO, EVALUACIÓN Y EJECUCIÓN DE UN PLAN LOCAL DE INMIGRACIÓN (CON ESPECIAL ATENCIÓN AL MUNICIPIO

DE $\boldsymbol{E} \boldsymbol{L} \boldsymbol{C H E}$ ). Navarra: Thomson Reuters, Aranzadi. 2013. 275 pp.

Esta obra es el resultado del trabajo de un colectivo de profesionales -vinculados a la intervención social con inmigrantes, desde una perspectiva local- bajo la coordinación del profesor Alfonso Ortega Giménez, quien cuenta con una dilatada experiencia en el estudio e investigación de temas migratorios y que, desde su puesto de gestión como Director del Observatorio de la Inmigración de la ciudad de Elche, ha podido llevar las riendas de este trabajo en colaboración con el Excelentísimo Ayuntamiento de Elche y las Universidades de la ciudad.

Sin duda, el objeto de desarrollo es clave para ordenar el trabajo de la corporación local respecto al tema migratorio. Poner sobre la mesa unas pautas de actuación para lograr una adecuada gestión de la inmigración es un punto fundamental para la administración de nuestros municipios, pero sobre todo, en aquellas ciudades que, como Elche, presentan una elevada presencia de extranjeros en su territorio.

En este contexto se nos muestra un resultado que pone frente al lector dos documentos: el primero, el estudio elaborado por el grupo de trabajo que estudió la realidad de la inmigración en Elche, y el segundo, el Plan Local de Inmigración que posteriormente fue aprobado, a la vista de dicho estudio. La finalidad de la obra es doble y se deja claramente planteada en el Prólogo de la misma: por un lado, dar a conocer el contenido del Plan Local de Inmigración a los ciudadanos del municipio, y por otro, proponer un modelo de trabajo que "sirva como referencia" para la elaboración de planes similares por otros municipios. Todo ello para que la puesta en práctica de un Plan Local de Inmigración pueda dar respuesta a los desafíos que presenta la diversidad cultural, esto es, la integración social y cultural de los nuevos ciudadanos que forman parte de la vida social española.

Para cumplir con ese objetivo, la obra se estructura en dos partes: la primera hace referencia a los fundamentos para la elaboración de un plan local de integración de la inmigración y la segunda recoge el modelo de plan local de integración aprobado para el municipio de Elche.

En relación con la primera, las aportaciones del estudio previo, éstas hacen referencia a las siguientes cuestiones: estudio del marco jurídico de la inmigración y la gestión de la diversidad; análisis de los rasgos característicos del plan, concepto y metodología de trabajo; estudio de los principios rectores y consideraciones previas en la redacción del plan; propuesta de objetivos generales y específicos; análisis de las medidas básicas y ejes estratégicos de actuación; y por último, la gobernanza del plan.

En la segunda parte se expone el modelo de plan local el cual comprende un estudio del panorama de la situación migratoria en municipio, para luego dar paso a las medidas específicas por área de actuación: acogida, información, orientación y asesoramiento; educación y cultura; empleo y promoción económica; sanidad. Por último, se recogen las medidas de carácter transversal que hacen referencia a temas claves de la vida local, tales como, igualdad de mujeres y hombres, participación $\mathrm{y}$ asociacionismo; convivencia y finalmente, inmigración, extranjería y nacionalidad.

El resultado de este trabajo colectivo es de una lectura cómoda para cualquier lector, avezado o no en temas de gestión migratoria. La lectura es en doble clave: pues el valor prácti- 
co está presente tanto para técnicos, como para usuarios y/o destinatarios finales de este plan; de ahí el carácter divulgativo de este libro. En definitiva es una lectura recomendable y encierra una vez más el buen hacer de un equipo de profesionales comprometidos con el estudio de estos temas, los que con cada una de sus aportaciones contribuyen a enriquecer el fondo bibliográfico nacional.

L. S. HEREDIA SÁNCHEZ

U. Miguel Hernández (Elche)

Kiko MORA y

Eduardo VIÑUELA (eds.)

ROCK AROUND SPAIN. HISTORIA, INDUSTRIA, ESCENAS Y MEDIOS DE COMUNICACIÓN. Lleida: Edicions de la Universitat de Lleida. 2013. 264 pp.

Este texto es una publicación que se ha elaborado con la colaboración conjunta de diferentes autores que pertenecen a diversos campos de especialización abarcando disciplinas como la sociología, la musicología, el periodismo, o la semiótica así como la participación de una serie de profesionales integrantes del engranaje de la industria musical en su vertiente más "rockera", ya sean agentes discográficos, críticos musicales, disc-jockeys o músicos profesionales. Todo este entramando multidisciplinar, sin embargo, encuentra su cauce en la finalidad que este libro ofrece en su conjunto, que no es otra que realizar una aproximación al ámbito del rock español desde una perspectiva ampliamente contrastada pero sólida en cada uno de sus apartados

Una primera sección histórica nos conduce por un amplio y detallado recorrido de los principales hitos musicales de la música rock en nuestro país, desde su entrada en la década de los años 50, pasando por su época dorada durante la década ochentera, en la que esta lectura se adentra en profundidad en el fenómeno sociocultural y mediático que supuso La Movida y finaliza este recorrido histórico remarcando las consecuencias que la música rock y pop ha ido afrontando en sus últimos veinte años de historia hasta finalizar la primera década del nuevo siglo. En todo momento se desarrolla cada una de las secciones junto con un detallado contexto político y social que nos muestra cómo se fue propiciando y asentando esta evolución en la trayectoria de este género en España en relación al momento histórico contextualizado que se vivía.

Un segundo apartado nos invita a adentrarnos en el entramado de la industria musical. Una aproximación que permite comprender cómo una sociedad que salía de una interminable etapa dictatorial se encontraba dividida, reprimida y esclava de un paupérrimo bagaje cultural que la situaba a la cola de la franja occidental europea. La llegada de la democracia o la entrada en la Comunidad Económica Europea, sirven de plataforma para que diversas escenas musicales comiencen a surgir en un entorno impensable años atrás, en el que se comienza a desatar una vorágine de movimientos que convergerán durante la década de 1980, sin duda la de mayor proliferación de grupos, corrientes y tendencias, ya no sólo musicales, también, ideológicas, reivindicativas o estéticas. Este torrente sociocultural nos permitirá comprender mejor el crecimiento abrumador que experimentó la industria musical en su totalidad, desde las discográficas más poderosas, hasta las alternativas musicales independientes que se abastecen de artistas que se nutren de nuevas influencias a finales de esta década y continuará su desarrollo durante y las siguientes.

Rock Around Spain además de explicar estos orígenes del rock de un modo cronológicamente ordenado, profundiza en las diferentes escenas musicales que coexisten, en diferentes áreas del marco geográfico español. Además de la incuestionable fuerza que ejercía la capital del Estado español sobre el crecimiento de 
los diferentes componentes de la industria musical, no sería la única, aunque que no cabe duda de que fue la plataforma principal de lanzamiento para los artistas emergentes, y el escenario pretendido por una serie innumerable de aspirantes a protagonizar una porción de la escena musical española del momento. No todo comenzará y acabará en Madrid y La Movida, como queda reflejado en las siguientes páginas el recorrido de las diferentes escenas detalla la escena del rock radikal vasco, continuando con los acontecimientos más significativos del rock en Cataluña, los aromas flamencos impregnados en la escena rockera andaluza así como el fenómeno desencadenado por la denominada Movida de Vigo en Galicia.

Como colofón, este libro sugiere una visión panorámica, esta vez con el punto de mira puesto en los diferentes medios de comunicación que han tenido un rol trascendente en la difusión de la música rock, bien sea a través de la prensa escrita, la radio, la televisión o el cine. El rock ha cohabitado en los diferentes medios bien gracias al trabajo, o bien esfuerzo altruista incondicional, de un conjunto de amantes del género que a toda costa trataron de aunar fuerzas para que no quedas relegado al olvido o la marginación, aunque es palpable que el rock en este país, es poseedor de una etiqueta más pequeña, que impidió el desarrollo que la propia inercia con la que irrumpía, había trazado. De todas formas, los medios de comunicación siguen ejerciendo un constante feed-back con el género rock, como se señala en el último apartado del libro; sigue presente en diferentes campañas publicitarias y a través de los spot. La publicidad y el rock continúan imbricados en una constante lucha por influir en los hábitos de consumo, y condicionar la conducta en busca del éxito.

$$
\begin{array}{r}
\text { P. BUIL TERCERO } \\
\text { U. Complutense (Madrid) }
\end{array}
$$

